\title{
Selected CC and CXC chemokines in children with atopic asthma
}

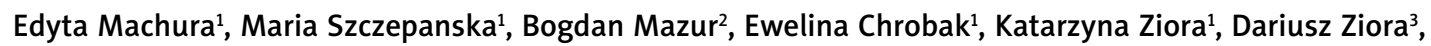 \\ Alicja Kasperska-Zajac ${ }^{4}$
}

${ }^{1}$ Department of Pediatrics, Medical University of Silesia, School of Medicine with the Division of Dentistry, Zabrze, Poland 2Department of Microbiology and Immunology, Medical University of Silesia, School of Medicine with the Division of Dentistry, Zabrze, Poland

${ }^{3}$ Chair and Department of Pulmonology and Tuberculosis, Medical University of Silesia, School of Medicine with the Division of Dentistry, Zabrze, Poland

${ }^{4}$ Chair and Clinical Department of Internal Diseases, Dermatology and Allergology, Medical University of Silesia, School of Medicine with the Division of Dentistry, Zabrze, Poland

Adv Dermatol Allergol 2016; XXXIII (2): 96-101

DOI: 10.5114/ada.2016.59150

\begin{abstract}
Introduction: There are only limited data on CC and CXC chemokines regulation in children with asthma.

Aim: We compared the serum profile of selected CC and CXC chemokines in patients with atopic asthma and healthy children.

Material and methods: Serum concentration of CC chemokines RANTES, MCP-1, and CXC chemokines IP-10, MIG, IL-8, RANTES was measured using cytometric bead array in 44 children with atopic asthma and 17 healthy subjects. Results: The concentration of RANTES was significantly higher and the MIG level was lower in all children with asthma as compared to their control counterparts. We observed increased RANTES and decreased MIG levels also in patients with stable asthma when compared with children in the control group. The IP-10 concentration was similar between the whole asthma group and healthy controls, while significantly increased levels of this chemokine in acute asthma have been observed when compared to stable asthma. For MCP- 1 and IL-8, the serum concentration was similar in all compared groups. The MIG concentration correlated positively with IP-10, IL-8, and CRP levels and negatively with the eosinophil count. A negative correlation between the IP-10 and eosinophil count and a negative correlation between FEV 1 and IP-10 were found.

Conclusions: An increased serum RANTES level in children with asthma may result in enhancement of Th2 lymphocyte recruitment into the airway. A decreased expression of Th1 chemokine MIG in children with stable asthma may contribute to a diminished antagonizing effect on Th2 cytokine production and hence intensify Th2 predominance. An increased IP-10 level in children during an asthma attack suggest that this chemokine is a serological marker of disease exacerbation.
\end{abstract}

Key words: atopic asthma, chemokines, RANTES, MIG, IP-10, IL-8, children.

\section{Introduction}

Allergic asthma is one of the most common diseases of childhood which results from both genetic and environmental factors. The characteristic lesion found in asthmatic airways is the allergic inflammation with overabundance of eosinophils, mast cells, and activated CD4+Th2 lymphocytes [1]. These cells release a broad range of inflammatory mediators including histamine, eicosanoids, cytokines, chemokines and growth factors [2].
There is growing evidence that chemokines contribute to the development and maintenance of allergic inflammation because at the allergic site they coordinate the recruitment and cells activation via their receptors, both leukocytes and residential cells [3]. For example, CC chemokine called the CCL5 regulated on activation, normal T cell expressed and secreted (RANTES) in conjunction with its receptor (CCR), which is preferentially expressed on Th2 cells, eosinophils or monocytes is involved in the

Address for correspondence: Maria Szczepanska MD, PhD, Department of Pediatrics, Medical University of Silesia, School of Medicine with the Division of Dentistry, 13/15 3 Maja St, 41-800 Zabrze, Poland, phone: +48 3237043 05, e-mail: dializy_dzieciece_zabrze@poczta.onet.pl

Received: 27.10.2014, accepted: 28.02.2015. 
pathogenesis of asthma [4, 5]. It is suggested that the Th2 predominant profile seen in asthma may result from a decreased production of Th1 related CXC chemokines and deficiency in the chemotaxis of Th1 cells and counter inhibition on Th2 cells chemoattraction $[6,7]$. However, recent studies questioned the opinion that Th1 cytokines' levels are decreased in asthma [8, 9]. In animal studies it has been reported that Th1 cells do not counterbalance but even worsen Th2 mediated airway inflammation [10]. Also during asthma exacerbation, both Th2 and Th1 cells increase in number in blood and induced sputum formation [11]. Although up-regulated expression of CC chemokines was documented in the airways and serum of asthmatic subjects in numerous studies, the role of CXC chemokines in asthma is lesser known [5]. There are only limited data on CC and CXC chemokines regulation in children with asthma $[12,13]$.

\section{Aim}

In this study we compared the serum profile of selected CC chemokines including RANTES, monocytes chemoattractant protein 1 (MCP-1) and CXC chemokines - interferon- $\gamma$-inducible protein-10 (IP-10/CXCL10), macrophage inflammatory proteins (MIG/CXCL9), interleukin 8 (IL-8) in patients with atopic asthma and healthy children, in order to investigate their role in pathogenesis of asthma and to evaluate their relationship with the severity of airway obstruction.

\section{Material and methods}

The study group was comprised of 44 children with atopic asthma and 17 healthy subjects. The diagnosis of asthma, assessment of severity, asthma management plan and asthma control level were established according to the GINA 2006 criteria. Thirty eight children aged $>6$ years underwent spirometric assessment and presented reversibility of airway obstruction, as documented by a positive response to a bronchodilator of at least $12 \%$ increased forced expiratory volume in $1 \mathrm{~s}\left(\mathrm{FEV}_{1}\right)$. All the children had positive skin prick test (SPT) results to $\geq 1$ allergens. A positive SPT was defined as a mean diameter of at least $3 \mathrm{~mm}$ in the presence of negative diluent and positive histamine controls.

Children with asthma were divided in two groups:

- stable asthma group: 29 children with well-controlled asthma (mean age: $11.4 \pm 1.0$, range: $5-16$ years) were recruited during their scheduled outpatient clinic visits. All patients had no asthma exacerbation and experienced no change in their treatment course for at least two months and were treated with a low dose of inhaled corticosteroids (ICS) (100-200 $\mu \mathrm{g} /$ day).

- children with exacerbation of asthma: this group consisted of 15 age-matched children (mean age: $9.9 \pm 1.0$, range: 4.5-16 years) hospitalized for asthma exacerbation. Before admission all the children experienced a change in
Table 1. Demographic and clinical characteristics of children with asthma and healthy controls

\begin{tabular}{lcc}
\hline Parameter & Asthma & Controls \\
\hline Age [years] & $10.5 \pm 0.5$ & $11 \pm 0.2$ \\
\hline M/F & $24 / 20$ & $10 / 7$ \\
\hline Total serum IgE $[\mathrm{IU} / \mathrm{ml}]$ & $335.2 \pm 47.6$ & $42.5 \pm 16.5^{*}$ \\
\hline $\mathrm{CRP}[\mathrm{mg} / \mathrm{l}]$ & $4.8 \pm 1.5$ & $3.1 \pm 0.8$ \\
\hline $\begin{array}{l}\text { Absolute number of blood } \\
\text { eosinophils, \% }(n, \text { cells/ } \mu \mathrm{l})\end{array}$ & $344.8 \pm 45.1$ & $144 \pm 17.5^{\star *}$ \\
\hline $\mathrm{FEV}_{1} \%$ predicted & $86.3 \pm 2.1$ & Not done \\
\hline FEV $/ \mathrm{FVC}$ & $86.3 \pm 1.7$ & \\
\hline FVC \% predicted & $86.3 \pm 1.9$ & \\
\hline
\end{tabular}

${ }^{*} p<0.001,{ }^{* *} p<0.05$. Data are shown as mean \pm standard error.

their treatment due to loss of asthma control (nebulized salbutamol, course of 1-3 day = systemic corticosteroid and/or increasing dose of ICS) during last 5 days.

The control group consisted of 17 healthy children (mean age: $11.01 \pm 0.2$, range: $4-16$ years) with a negative history of allergic diseases, normal level of total serum IgE and negative results of skin prick test to a panel of aeroallergens (dust mite, mixed grass or tree pollen, cat and dog; Allergopharma, Reinbeck, Germany). Children included into the control group attended the outpatient pediatric clinic for non-immunological, non-inflammatory health problems and needed venous puncture.

The characteristics of study groups are summarized in Table 1.

The present study was approved by the Ethics Committee of the Medical University of Silesia in Katowice and written informed consent was obtained from children's parents.

Blood samples from children with exacerbation or stable asthma and healthy children were collected at the time of admission to hospital or during the scheduled clinic visit and serum samples were stored at $-45^{\circ} \mathrm{C}$ until assayed.

\section{Assays for chemokines and cytokines in serum}

The concentrations of IP-10, MIG, IL-8, MCP-1 and RANTES in serum were measured simultaneously using human chemokine cytometric bead array (CBA) reagent (Becton Dickinson Biosciences Pharmingen, CA). Samples were analyzed on a multifluorescence BD FACSCaliburTM flow cytometer using BD CellQuestTM software and BDTM CBA Software. The assay sensitivities of these five chemokines were 2.8, 2.5, 2.7, 1.0, and $0.2 \mathrm{pg} / \mathrm{ml}$, respectively. The coefficients of variation of all chemokine assays were less than $10 \%$.

\section{Statistical analysis}

Statistical analysis was performed using software package (Statistica, version 9.0) and data presented as 


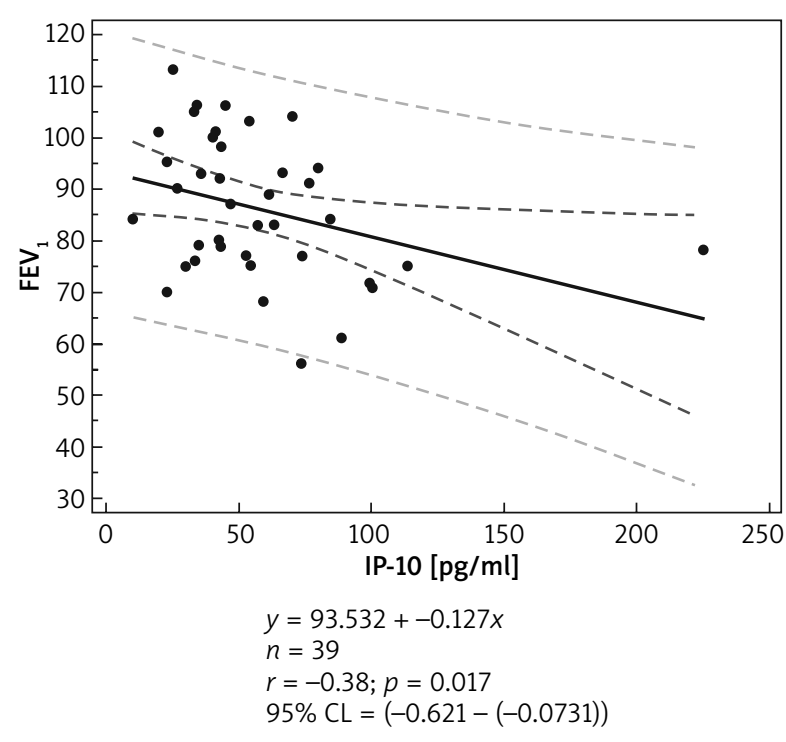

Figure 1. Negative correlation between $\mathrm{FEV}_{1}$ and serum MIG concentration in asthmatic children

mean values \pm SE. Kruskal-Wallis and Mann-Whitney $U$ tests were used for comparisons between groups. Correlations between variables were tested using Spearman's test. $P$-values less than 0.05 were considered statistically significant.

\section{Results}

Chemokines RANTES/CCL5, MIG/ CXCL9 and IP-10/ CXCL10 were abundant in all the samples. Chemokines MCP-1 and IL-8 were detectable in $62.3 \%$ and $83.6 \%$ of samples, respectively. The MCP was abundant in 27 children with asthma (61.4\%, 17 stable, 10 unstable) and 11 control subjects (64\%). Interleukin 8 was abundant in 39 children with asthma (88.6\%, 25 stable, 14 unstable) and 12 control subjects (70.5\%).

The concentration of CC chemokine RANTES was significantly higher in all children with asthma as compared to their control counterparts. We observed an increased RANTES level also in patients with stable asthma when compared with children in control group. CXC chemokine MIG was significantly lower in all children with asthma. A significantly decreased level of this chemokine was seen also in children with stable asthma as compared to healthy children. IP-10 concentration was similar in the whole asthma group and healthy controls, while significantly increased levels of this chemokine in acute asthma have been observed when compared to stable asthma.

For MCP-1 and IL-8, plasma concentrations were similar between all compared groups (Table 2).

\section{Correlation between chemokines and laboratory parameters}

Chemokine MIG concentration correlated positively with IP-10, IL-8, and CRP levels ( $r=0.56, p=0.001$, $r=0.37, p=0.01, r=0.39, p=0.01$, respectively) and negatively with eosinophil count $(r=-0.457, p<0.057)$ (Figure 1).

On the other hand a negative correlation between IP-10 and eosinophil count $(r=-0.34, p<0.045)$ was detected. Moreover, a negative correlation between $\mathrm{FEV}_{1}$ and IP-10 ( $r=-0.38, p<0.05)$ was found (Figure 2$)$.

\section{Discussion}

In this study we aimed to characterize a selected Th1 and Th2 chemokine serum profile in patients with stable asthma and those who lost control of the disease and compare it with non-atopic healthy controls. Among examined chemokines, a serum level of RANTES was observed to be increased in children with stable, persistent asthma, suggesting an increased infiltration of inflammatory cells at the tissue level. RANTES plays an important role in attracting eosinophils, mast cells and basophils, macrophages/monocytes and lymphocytes T (preferentially of the Th2 type) to the asthmatic airways. In current literature, an elevated concentration of RANTES MRNA in bronchial biopsies [14] and increased RANTES levels in bronchoalveolar lavage (BAL) [15-17], exhaled breath condensate (EBC) [18], induced sputum [19], and serum or plasma [20-22] were reported by various authors.

Table 2. Serum chemokine levels in children with asthma and healthy controls

\begin{tabular}{|c|c|c|c|c|c|c|}
\hline Variable & $N$ & $\begin{array}{c}\text { IL-8 } \\
{[\mathrm{pg} / \mathrm{ml}]}\end{array}$ & $\begin{array}{l}\text { RANTES } \\
{[\mathrm{pg} / \mathrm{ml}]}\end{array}$ & $\begin{array}{c}\text { MCP-1 } \\
{[\mathrm{pg} / \mathrm{ml}]}\end{array}$ & $\begin{array}{c}\text { MIG } \\
{[\mathrm{pg} / \mathrm{ml}]}\end{array}$ & $\begin{array}{c}\text { IP-10 } \\
{[\mathrm{pg} / \mathrm{ml}]}\end{array}$ \\
\hline \multicolumn{7}{|l|}{ Asthma: } \\
\hline All children & 44 & $6.8 \pm 1.2$ & $7595.9 \pm 160.9$ & $57.18 \pm 8.5$ & $217.0 \pm 56.1$ & $60.0 \pm 6.7$ \\
\hline Stable & 29 & $7.7 \pm 1.8$ & $7653.0 \pm 185.4^{\star *}$ & $58.5 \pm 10.9$ & $231.6 \pm 84.5^{\# \#}$ & $52.3 \pm 7.5$ \\
\hline Unstable & 15 & $5.1 \pm 0.8$ & $7485.6 \pm 314.7$ & $54.5 \pm 14.0$ & $188.8 \pm 25.7$ & $74.8 \pm 12.7^{8}$ \\
\hline Healthy children & 17 & $4.2 \pm 0.8$ & $6565.7 \pm 370.9^{\star}$ & $58.4 \pm 13.4$ & $329.3 \pm 75.0^{\#}$ & $83.3 \pm 14.5$ \\
\hline
\end{tabular}

${ }^{*}$ All children with asthma (stable and unstable) vs. control, $p<0.01$; ${ }^{* *}$ children with stable asthma vs. control, $p=0.01 ; *$ all children with asthma (stable and unstable) vs. control, $p<0.04 ;{ }^{\#}$ children with stable asthma vs. control, $p<0.02 ;{ }^{\&}$ children with unstable asthma vs. children with stable asthma, $p<0.05$. Data are shown as mean \pm standard error. 
Only a few studies assessed a serum RANTES concentration in asthmatic children and their results were to some extent different from findings obtained in adult patients with asthma.

In our present study we have found that a significantly increased RANTES level was seen in children with stable asthma, but not in an acute asthma attack as compared to controls. Similarly to our results, no significant difference in the serum RANTES level between asthmatic children with an acute asthma attack and controls was observed in other studies [12, 20, 23]. However, significantly increased serum RANTES levels measured a few weeks after the acute attack were observed by Reisli et al. Based on this study, authors thought that higher levels of this chemokine detected in asymptomatic asthmatic children after an acute attack may be an indicator of a chronic asthmatic inflammation. In turn, the RANTES level in asthmatic children with an acute attack of the disease, which was found to be lower than in stable asthmatic children, may result from the recruitment of RANTES into the asthmatic airways. Previous studies performed after exercising a challenge in children with asthma, in which an exhaled RANTES level was increased [24], and a circulating level of this chemokine was decreased [25] may support this concept. Additionally, in adult patients with delayed asthmatic response after the allergen challenge, a decreased serum concentration of CCL5 was observed [26].

An increased serum RANTES level in adult patients with stable asthma, irrespective of ICS usage was also observed in one study [19]. In children although the RANTES level was higher did not reach significance in comparison to healthy subjects $[12,20]$.

We did not find any correlation between RANTES and eosinophil count or spirometric parameters. In contrast to our results, in adult patients with an asthma positive correlation with severity of asthma [6, 18, 21], an absolute eosinophil count, serum IgE level [21] and percentage of sputum eosinophils [19] or percentage of eosinophils in bronchial mucosa [14] and RANTES was observed.

Notably, no correlation between the elevated level of RANTES and eosinophils in BAL fluid in children with asthma was found [17].

In our study, the serum level of MCP-1 was similar in children with asthma irrespective of current control of disease symptoms and in healthy ones. We have chosen this marker as MCP-1 may play a significant role in asthma being involved in the chemoattraction of monocytes, lymphocytes and basophils into tissues - all of the cells characteristic of allergic inflammation. However, results from different studies were apparently conflicting.

An increased expression of MCP-1 in BAL $[15,16,27]$ and a higher MCP-1 level in serum [6] and in saliva of asthmatic adults has been confirmed [28]. On the contrary, Folkard et al. did not find an increased expression MCP-1 in BAL of asthmatic adult patients [29]. Regarding

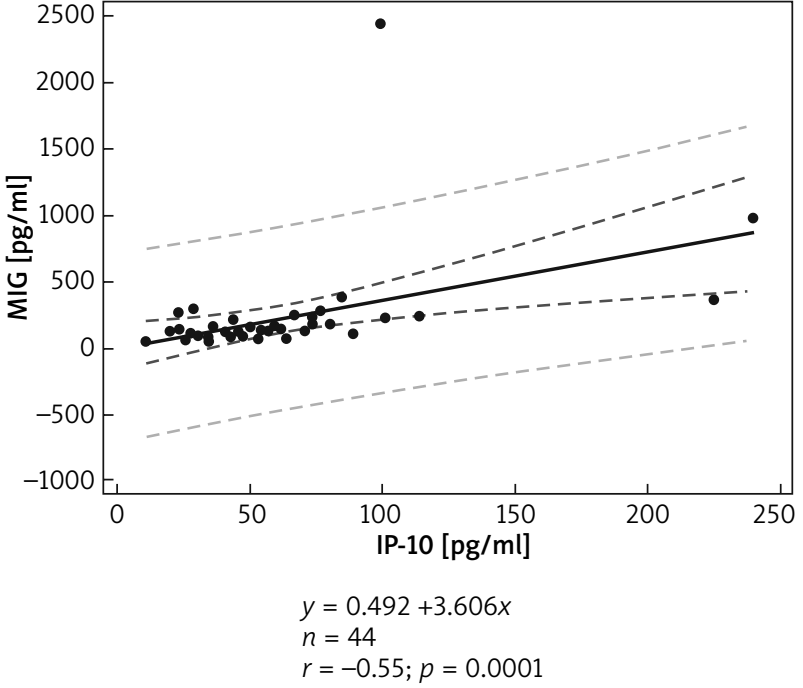

Figure 2. Positive correlation between MIG and IP-10 serum concentration in children with asthma

the studies in children with asthma in a study by Giuffrida et al., a serum MCP-1 level was increased [22]. In another study, MCP-1 was elevated only in acute course, but not in stable asthma [12].

Although most studies in asthma revealed an upregulated expression of Th2 cytokines at the site of allergic inflammation, recent reports suggest that Th1-type response also plays an important role in the pathogenesis of asthma. Both INF- $\gamma$ - inducible protein of $10 \mathrm{kDA}$ (IP-10) and monokine induced by INF- $\gamma$ (MIG) belong to the CXC chemokine subfamily, members of which interact with the common receptor CXCR3 that is highly expressed on Th0, Th1 and NK lymphocytes and to a lesser extent on eosinophils and play an important role in the Th1 mediated immunoresponse $[4,5]$. The functional role of Th1 associated chemokines in asthma remains still controversial. In an animal model of this asthma, Th1 associated chemokine can both downregulate [30, 31] and augment $[32,33]$ the Th2-type of airways inflammation, which was previously demonstrated by different authors. Results of human studies suggest that Th1-related CXC chemokine IP-10 may be a useful inflammatory marker of asthma exacerbation [18, 34, 35]. IP-10 was detected in higher concentrations in BAL of asthmatic children as compared to control subjects [36]. Moreover, a plasma level of IP-10 was significantly higher in children during an acute asthma attack than during a subsequent convalescent period and in children with stable asthma [13]. In a higher concentration IP-10 was detectable in sputum, EBC [34] and saliva [28] in asthmatic patients during exacerbation. On the other hand, an IP-10 concentration was elevated also in BAL of patients with stable asthma and its mRNA and protein expression was increased in the bronchial mucosa as compared with healthy controls [27]. 
On the contrary, in another study, lower serum IP-10 levels $[6,7]$ and a decreased production of IP-10 by peripheral blood mononuclear cells (PBMC) activated by mitogen, allergen or cytokines was demonstrated in asthmatic patients [6, 37].

In the present study we did not find any difference in the serum IP-10 level between children with asthma and control subjects, but in children with asthma exacerbation, the level of IP-10 was significantly higher compared with counterparts with stable asthma and this finding is consistent with previous study conducted in asthmatic children [12]. It is believed that in a clinical setting, > 80\% asthma exacerbations are induced by viral infection, in particular by the rhinovirus. The presence of an increased serum IP-10 level was highly specific to acute virus-induced asthma exacerbations [35]. In this study we did not perform virological examination, but all children with the unstable asthma had a history of a respiratory tract infection with an increased CRP level prior to exacerbation.

Recent data demonstrated that IP-10 can directly upregulate the effector function of eosinophils such as adhesion and production of cytokines/chemokines. This effect might be involved in the activation and infiltration of eosinophils in the airway of asthma, especially in virus induced asthma [38]. We confirmed that IP-10 negatively correlated with $\mathrm{FEV}_{1}[18,35,39]$.

In contrast to IP-10, the role of MIG in the pathogenesis of asthma has not been fully elucidated. The IFN- $\gamma$ inducible MIG significantly reduces airway hyperresponsiveness and eosinophil accumulation in animal models of allergen challenge. MIG diminishes IL-4 and enhances IL-12 levels, directing activated T cells toward a Th1 phenotype [30]. In a study by Lai et al., a plasma level of MIG was significantly higher in children during an acute asthma attack than during a subsequent convalescent period and comparing with children with stable asthma [13]. In another study, the plasma level of MIG did not differ between steroid naïve children, children on ICS and the control group, but the ex vivo release of MIG was attenuated in PBMC activated with allergen, mitogen and IL-18 [6].

In the study of Hartl et al., the expression of MIG in BAL in children with asthma was higher as compared to healthy controls [36]. In contrast, in adults with a severe asthma expression of this chemokine in BAL was lower than in mild asthmatic patients [40].

In our study, a circulating level of MIG was lower in asthmatic children than in controls, specifically in stable asthma. We also found that both MIG and IP-10 negatively correlated with the eosinophil number. As mentioned above, eosinophils expressing CXCR3 and IP-10 and MIG are capable of inducing eosinophilic chemotaxis and degranulation [33]. On the other hand, in a murine model of asthma MIG inhibited eosinophil recruitment to the lung $[30,41]$. The inhibition of eosinophils may be regarded as an endogenous mechanism of limiting the immune re- sponse and lung injury [41]. The MIG concentration in our study correlated with IL-8 and CRP levels.

The IL-8 or CXCL8 is a potent chemoattractant for neutrophils, involved in airway inflammation in patients with asthma and associated with severity of asthma. But in our study we did not find any difference between the examined group and healthy children.

There are several limitations of our study, which should be considered when interpreting the study results. The study group was rather small, which reduced the power of the study. We assessed only the peripheral blood of the subjects without concomitant assessment of the airway by the invasive method (BAL, biopsy, EBC, induced sputum). This was a cross-sectional study. Finally, all children were on ICS and results may be influenced by ICS.

\section{Conclusions}

In this study we demonstrated increased serum RANTES levels in children with asthma, which may result in a possible enhancement in Th2 lymphocyte recruitment into the airway. Moreover, a decreased expression of Th1 chemokine MIG in children with stable asthma may also contribute to the diminished antagonizing effect on Th2 cytokine production and hence Th2 predominance. Since the IP-10 level increased in children during an asthma attack it seems that this chemokine may be a serological marker of disease exacerbation.

\section{Acknowledgments}

This work was supported by grant 109/K/3/0 from the Medical University of Silesia in Katowice, Poland.

\section{Conflict of interest}

The authors declare no conflict of interest.

\section{References}

1. Rogala B, Bozek A, Gluck J, Jarzab J. Prevalence of IgE-mediated allergy and evaluation of Th1/Th2 cytokine profiles in patients with severe bronchial asthma. Postep Derm Alergol 2015; 32: 274-80.

2. Cohn L, Elias JA, Chupp GL. Asthma: mechanisms of disease persistence and progression. Annu Rev Immunol 2004; 22: 789-815.

3. Nedoszytko B, Sokołowska-Wojdyło M, Ruckemann-Dziurdzińska K, et al. Chemokines and cytokines network in the pathogenesis of the inflammatory skin diseases: atopic dermatitis, psoriasis and skin mastocytosis. Postep Derm Alergol 2014; 31: 84-91.

4. Bisseta LR, Schmid-Grendelmeier P. Chemokines and their receptors in the pathogenesis of allergic asthma: progress and perspective. Curr Opin Pulm Med 2005; 11: 35-42.

5. Borish LC, Steinke JW. Cytokines and chemokines. J Allergy Clin Immunol 2003; 111 (2 Suppl.): S460-75. 
6. Lun SW, Wong CK, Ko FW, et al. Aberrant expression of CC and CXC chemokines and their receptors in patients with asthma. J Clin Immunol 2006; 26: 145-52.

7. Ko FW, Lun SW, Wong CK, et al. Decreased T-bet expression and changes in chemokine levels in adults with asthma. Clin Exp Immunol 2007; 147: 526-32.

8. Lloyd CM, Hessel EM. Functions of T cells in asthma: more than just Th2 cells. Nat Rev Immunol 2010; 10: 838-48.

9. Cho SH, Stanciu LA, Holgate ST, Johnston SL. Increased interleukin-4, interleukin-5, and interferon-gamma in airway CD4+ and CD8+ T cells in atopic asthma. Am J Respir Crit Care Med 2005; 171: 224-30.

10. Hansen G, Berry G, DeKruyff RH, Umetsu DT. Allergen-specific Th1 cells fail to counterbalance Th2 cell-induced airway hyperreactivity but cause severe airway inflammation. J Clin Invest 1999; 103: 175-83.

11. Mamessier E, Nieves A, Lorec AM, et al. T-cell activation during exacerbations: a longitudinal study in refractory asthma. Allergy 2008; 63: 1202-10.

12. Kato M, Yamada Y, Maruyama K, Hayashi Y. Serum eosinophil cationic protein and 27 cytokines/chemokines in acute exacerbation of childhood asthma. Int Arch Allergy Immunol 2010; 152 Suppl. 1: 62-6.

13. Lai ST, Hung CH, Hua YM, et al. T-helper 1-related chemokines in the exacerbation of childhood asthma. Pediatr Int 2008; 50: 99-102.

14. Powell N, Humbert M, Durham SR, et al. Increased expression of MRNA encoding RANTES and MCP-3 in the bronchial mucosa in atopic asthma. Eur Respir J 1996; 9: 2454-60.

15. Alam R, York J, Boyars M, et al. Increased MCP-1, RANTES, and MIP-1alpha in bronchoalveolar lavage fluid of allergic asthmatic patients. Am J Respir Crit Care Med 1996; 153: 1398-404.

16. Tillie-Leblond I, Hammad H, Desurmont S, et al. CC chemokines and interleukin-5 in bronchial lavage fluid from patients with status asthmaticus. Potential implication in eosinophil recruitment. Am J Respir Crit Care Med 2000; 162: 586-92.

17. Rojas-Ramos E, Avalos EAF, Perez-Fernandez L, et al. Role of the chemokines RANTES, monocyte chemotactic proteins -3 and -4 , and eotaxins -1 and -2 in childhood asthma. Eur Respir J 2003; 22: 310-6.

18. Matsunaga K, Ichikawa T, Yanagisawa S, et al. Clinical application of exhaled breath condensate analysis in asthma: prediction of FEV1 improvement by steroid therapy. Respiration 2009; 78: 393-8.

19. Kim CK, Choi J, Callaway Z, et al. Increases in airway eosinophilia and a Th1 cytokine during the chronic asymptomatic phase of asthma. Respir Med 2010; 104: 1436-43.

20. Reisli I, Dogu EF, Ikincigullari KA, Babacan E. Evaluation of serum RANTES levels in childhood asthma. Asthma Allergy Immunol 2012; 10: 78-83.

21. Saad-El-Din Bessa S, Abo El-Magd GH, Mabrouk MM. Serum chemokines RANTES and monocyte chemoattractant protein-1 in Egyptian patients with atopic asthma: relationship to disease severity. Arch Med Res 2012; 43: 36-41.

22. Giuffrida MJ, Valero N, Mosquera J, et al. Increased cytokine/ chemokines in serum from asthmatic and non-asthmatic patients with viral respiratory infection. Influenza Other Respir Viruses 2014; 8: 116-22.

23. Hsieh KH, Chou CC, Chiang BL. Immunotherapy suppresses the production of monocyte chemotactic and activating factor and augments the production of IL-8 in children with asthma. J Allergy Clin Immunol 1996; 98: 580-7.
24. Keskin O, Keskin M, Kucukosmanoglu E, et al. Exhaled RANTES and interleukin 4 levels after exercise challenge in children with asthma. Ann Allergy Asthma Immunol 2012; 109: 303-8.

25. Boznański A, Rudzka D. The level of RANTES and interleukin-8 in serum of children with bronchial asthma after an exercise test. Pneumonol Alergol Pol 1998; 66: 148-53.

26. Pelikan Z. Chemokine profiles in blood associated with delayed asthmatic response to allergen challenge. Respir Med 2013; 107: 47-59.

27. Miotto D, Christodoulopoulos P, Olivenstein R, et al. Expression of IFN-gamma-inducible protein; monocyte chemotactic proteins 1, 3 and 4; and eotaxin in Th1- and Th2-mediated lung diseases. J Allergy Clin Immunol 2001; 107: 664-70.

28. Little FF, Delgado DM, Wexler PJ, et al. Salivary inflammatory mediator profiling and correlation to clinical disease markers in asthma. PLoS One 2014; 9: e84449.

29. Folkard SG, Westwick J, Millar AB. Production of interleukin-8, RANTES and MCP-1 in intrinsic and extrinsic asthmatics. Eur Respir J 1997; 10: 2097-104.

30. Thomas MS, Kunkel SL, Lukacs NW. Regulation of cockroach antigen-induced allergic airway hyperreactivity by the CXCR3 ligand CXCL9. J Immunol 2004; 173: 615-23.

31. Wiley R, Palmer K, Gajewska B, et al. Expression of the Th1 chemokine IFN-gamma-inducible protein 10 in the airway alters mucosal allergic sensitization in mice. J Immunol 2001; 166: 2750-9.

32. Medoff BD, Sauty A, Tager AM, et al. IFN-gamma-inducible protein 10 (CXCL10) contributes to airway hyperreactivity and airway inflammation in a mouse model of asthma. J Immunol 2002; 168: 5278-86.

33. Jinquan $\mathrm{T}$, Jing $\mathrm{C}$, Jacobi $\mathrm{HH}$, et al. CXCR3 expression and activation of eosinophils: role of IFN-gamma-inducible protein-10 and monokine induced by IFN-gamma. J Immunol 2000; 165: 1548-56.

34. Warwick G, Thomas PS, Yates DH. Non-invasive biomarkers in exacerbations of obstructive lung disease. Respirology 2013; 18: 874-84.

35. Wark PA, Bucchieri F, Johnston SL, et al. IFN-gamma-induced protein 10 is a novel biomarker of rhinovirus-induced asthma exacerbations. J Allergy Clin Immunol 2007; 120: 586-93.

36. Hartl D, Griese M, Nicolai T, et al. Pulmonary chemokines and their receptors differentiate children with asthma and chronic cough. J Allergy Clin Immunol 2005; 115: 728-36.

37. Wada K, Kobayashi T, Matsuwaki Y, et al. Alternaria inhibits double-stranded RNA-induced cytokine production through Toll-like receptor 3. Int Arch Allergy Immunol 2013; 161 Suppl. 2: 75-83.

38. Takaku Y, Nakagome K, Kobayashi T, et al. IFN-gamma-inducible protein of $10 \mathrm{kDa}$ upregulates the effector functions of eosinophils through beta2 integrin and CXCR3. Respir Res 2011; 12: 138-43.

39. Rojas-Dotor S, Segura-Méndez NH, Miyagui-Namikawa K, Mondragón-González R. Expression of resistin, CXCR3, IP10, CCR5 and MIP-1alpha in obese patients with different severity of asthma. Biol Res 2013; 46: 13-20.

40. Brasier AR, Victor S, Boetticher G, et al. Molecular phenotyping of severe asthma using pattern recognition of bronchoalveolar lavage-derived cytokines. J Allergy Clin Immunol 2008; 121: 30-7.

41. Fulkerson PC, Zimmermann N, Brandt EB, et al. Negative regulation of eosinophil recruitment to the lung by the chemokine monokine induced by IFN-gamma (Mig, CXCL9). Proc Natl Acad Sci USA 2004; 101: 1987-92. 